\title{
Incremental load training improves renal fibrosis by regulating the TGF-ß31/TAK1/MKK3/p38MAPK signaling pathway and inducing the activation of autophagy in aged mice
}

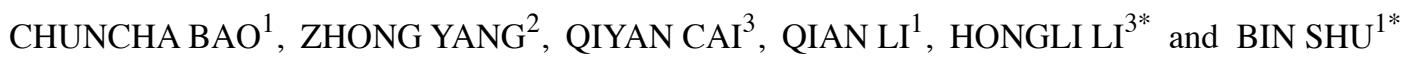 \\ ${ }^{1}$ Department of Rehabilitation Medicine, University Town Hospital, Chongqing Medical University, Chongqing 401331; \\ ${ }^{2}$ Department of Clinical Blood Teaching and Research; ${ }^{3}$ Department of Histology and Embryology, \\ Army Medical University, Chongqing 400038, P.R. China
}

Received April 19, 2019; Accepted July 26, 2019

DOI: $10.3892 / \mathrm{ijmm} .2019 .4344$

\begin{abstract}
Recent studies have confirmed that kidney tissue fibrosis is closely linked to the natural aging of organs. One of its major characteristics is the reduction of autophagic activity. However, to date, few studies have assessed whether incremental load training is able to improve the occurrence of renal fibrosis caused by natural aging and the underlying mechanisms. In the present study involving male C57/BL mice, an elderly exercise group (OY group) was subjected to progressive load-increasing rotary-bar training (5 days/week, lasting for 6 weeks), with an elderly control group (OC group) and a young control group (YC group) used as controls. Renal fibrosis and autophagy-associated indicators were assessed by Masson's staining, reverse transcription-quantitative PCR analysis, western blotting, immunofluorescence and transmission electron microscopy. The results suggested that collagen deposition in the basal part of the renal tubular epithelium and glomeruli in the OY group was significantly lower than that in the OC group. In the OC group, the protein expression levels of E-cadherin, Beclin 1 and light chain 3 were significantly decreased, and increases in $\alpha$-smooth muscle actin-positive signals were observed in the glomerular matrix and renal capsule wall. Furthermore, the expression
\end{abstract}

Correspondence to: Professor Bin Shu, Department of Rehabilitation Medicine, University Town Hospital, Chongqing Medical University, 55 Daxuecheng Middle Road, Chongqing 401331, P.R. China

E-mail: shubin1017@163.com

Professor Hongli Li, Department of Histology and Embryology, Army Medical University, 30 Gaotanyan Street, Chongqing 400038, P.R. China

E-mail: lihongli@tmmu.edu.cn

*Contributed equally

Key words: aging, renal fibrosis, incremental load training, TGF-31/TAK1/MKK3/p38MAPK, autophagy of transforming growth factor (TGF)- $\beta 1$ and its downstream signaling molecules TGF- $\beta$-activated kinase 1 (TAK1), mitogen-activated protein kinase (MAPK) kinase (MKK3) and p38MAPK were downregulated following training. The present study confirmed that incremental load training may improve renal fibrosis in aged mice by regulating the TGF- $\beta 1 / \mathrm{TAK} 1 / \mathrm{MMK} 3 / \mathrm{p} 38 \mathrm{MAPK}$ signaling pathway and inducing the activation of autophagy to reduce the synthesis of extracellular matrix and delay the epithelial-mesenchymal transition. The present study provides a novel experimental basis for the intervention of incremental load training to prevent senile renal fibrosis.

\section{Introduction}

Aging has become a research interest in the field of medicine. Aging is an unavoidable process in the increase of lifespan. In general, the functions of various organs of the body exhibit gradual decreases in this process. The kidney, as an active metabolic and major excretory organ, is one of the organs to experience rapid tissue aging, which is frequently accompanied by renal fibrosis $(1,2)$. Studies have indicated that exercise is an important means of rehabilitation, which may effectively improve myocardial fibrosis (3). Whether exercise is able to improve the renal fibrosis caused by natural aging requires further investigation.

A large number of studies have suggested that aging of the kidneys is accompanied by interstitial fibrosis (4), which mainly manifests as an accumulation of extracellular matrix (ECM). The epithelial-mesenchymal transition (EMT) of renal tubular epithelial cells is considered to be one of the major reasons for the marked accumulation of $\operatorname{ECM}(5,6)$. EMT occurs in renal tubular epithelial cells and induces changes in epithelial surface markers, including E-cadherin and $\alpha$-smooth muscle actin ( $\alpha$-SMA), a myofibroblast surface marker of the tubulointerstitium (7).

Autophagy is a catabolic degradation system used to digest the unnecessary or damaged components of a cell. The decrease of autophagic activity caused by aging is closely linked to the occurrence and development of fibrosis (8). Previous studies have shown that transforming growth 
factor (TGF)- $\beta 1$ is the most critical factor in promoting the occurrence of EMT $(9,10)$. In addition, TGF- $\beta 1$ serves an important role in regulating autophagy. Ding et al (11) found that the deletion of light chain (LC)3B [LC3(-/-)] resulted in increased collagen deposition and increased mature profibrotic factor TGF- $\beta$ levels in the obstructed kidneys of mice. Beclin 1 heterozygous (Beclin 1+/-) mice also exhibited increased collagen deposition in the obstructed kidneys after UUO. Signaling downstream of the TGF- $\beta$ receptor complexes is regulated by the Smads family, a canonical pathway $(12,13)$. TGF- $\beta 1$ signaling via the non-Smads pathways is also involved in the development of fibrosis. Previous reports have demonstrated that TGF- $\beta 1$-activated kinase 1 (TAK1), a member of the mitogen-activating protein (MAP) kinase kinase (MKK) kinase family, is involved in TGF- $\beta$ signaling in the non-canonical pathway $(14,15)$. The decreased autophagic activity of the ECM is closely linked to the occurrence and development of fibrosis, and in a fibrosis model, the expression levels of autophagic proteins Beclin 1 and LC3 were decreased (16). Furthermore, Kim et al (17) indicated the involvement of the TGF- $\beta 1 /$ TAK1/MKK3/p38MAPK signaling pathway in the induction of autophagy. Therefore, it may be hypothesized that TGF- $\beta 1$ improves renal fibrosis by regulating the TAK1/MKK3/p38MAPK signaling pathway and inducing autophagic activation.

A number of basic and clinical studies have shown that exercise is able to delay the aging of skeletal muscle and brain tissue $(18,19)$ and improve cardiopulmonary exercise function. Therefore, the purpose of the present study was to subject aged mice to incremental load training, to compare and observe whether such incremental load training leads to an improvement of renal fibrosis in aged mice, and to further clarify whether the underlying mechanisms include the TGF- $\beta 1 / \mathrm{TAK} 1 / \mathrm{MMK} 3 / \mathrm{p} 38 \mathrm{AMPK}$ signaling pathway and induction of autophagic activation. The results may provide an experimental basis for the development of novel interventions to prevent and treat renal fibrosis.

\section{Materials and methods}

Experimental animals. A total of 36-healthy male C57/BL mice (19-month-old, n=24; weight, 26-28 g; and 2-month-old, $n=12$; weight, 14-16 g) were purchased from the Laboratory Animal Breeding and Research Center, Army Medical University (Chongqing, China; license no. SYXK-PLA-20170002). All surgical and care procedures were approved by the Laboratory Animal Welfare and Ethics Committee of the Third Military Medical University (Chongqing, China). All mice were housed in cages in a constant environment with $55 \pm 10 \%$ humidity, a temperature of $20 \pm 5^{\circ} \mathrm{C}$ and a 12-h light/dark cycle. Food and water were provided ad libitum.

Animal grouping and exercise training. The 36 healthy male C57/BL mice were divided into three groups: Young control group (YC group, $n=12$ ), elderly control group (OC group, $\mathrm{n}=12$ ) and elderly exercise group (OY group, $n=12)$. The OC group received free food and water. Exercise training was performed 5 days/week in the same room in which the animals were housed, for a duration of 6 weeks in which the load was gradually increased (low-intensity activity, referring to a movement speed $\leq 10 \mathrm{~m} / \mathrm{min}$, and exercise intensity of $30-40 \%$ $\left.\mathrm{VO}_{2} \max \right)(20,21)$. At the end of the exercise training period, the mice were sacrificed by cervical dislocation. Certain kidney tissues were fixed with neutral formaldehyde and the remaining tissues were rapidly frozen in liquid nitrogen. The exercise protocol is shown in Table I (22).

Reverse transcription-semiquantitative PCR (RT-sqPCR) analysis. The synthesis of primers was performed by Shanghai Bioengineering Co, Ltd. The purity and concentration of RNA were detected with a nucleic acid protein analyzer following extraction with TRIzol reagent. The RT of RNA into complementary (c)DNA was performed (cat. no. T2240; Beijing Solarbio Science \& Technology Co., Ltd.) at room temperature. The total volume of the PCR mixture was $25 \mu \mathrm{l}$, which consisted of $15 \mu \mathrm{l}$ 2X GO Tap Green Mix (Takara Bio, Inc.), $2 \mu \mathrm{l}$ forward primer, $2 \mu \mathrm{l}$ reverse primer, $1 \mu \mathrm{l} \mathrm{cDNA}$ and $5 \mu \mathrm{l}$ DEPC. The PCR amplification protocol was as follows: Pre-denaturation at $95^{\circ} \mathrm{C}$ for $5 \mathrm{~min}$, denaturation at $95^{\circ} \mathrm{C}$ for $30 \mathrm{sec}$ and renaturation at $55^{\circ} \mathrm{C}$ for $30 \mathrm{sec}$ ( 35 cycles) (C1000 Touch $^{\mathrm{TM}}$ Thermal Cycler; Bio Rach Laboratories, Inc.). Amplification products were separated via agarose gel electrophoresis (Sigma-Aldrich; Merck KGaA). Image-Pro Plus 6.0 image analysis software (Media Cybernetics, Inc.) was used to automatically analyze the images and calculate the gray scale values. The sequences of the primers of the target genes were as follows: Beclin 1, forward 5'-ATGGAGGGGTCT AAGGCGTC-3' and reverse 5'-TGGGCTGTGGTAAGTA ATGA-3'; LC3II forward 5'-GACCGCTGTAAGGAGGTG-3' and reverse 5'-AGAAGCCGAAGGTTTCTTG-3'; $\beta$-actin, forward primer 5'-GTGACGTTGACATCCGTA-3' and reverse 5'-GTAACAGTCCGCCTA-3'.

Masson's staining. The 4- $\mu$ m-thick kidney tissue slices were dehydrated through a graded series of ethanol. According to the protocol of the Masson's staining kit (cat. no. G1340; Beijing Solarbio Science and Technology, Co., Ltd.), the procedure was as follows: Tissues were stained with Weigert's hematoxylin in acid solution for $5 \mathrm{~min}$, replaced with Masson's blue solution and incubated for $3 \mathrm{~min}$, followed by rinsing in distilled water for $1 \mathrm{~min}$, staining for $5 \mathrm{~min}$ with Ponceau acid fuchsin, differentiation in phosphomolybdic acid solution for $2 \mathrm{~min}$, rinsing in distilled water for $1 \mathrm{~min}$, immediately stained with aniline blue solution for 5 min and mounted with neutral gum sealant (all steps at room temperature). Pathological changes in the kidney tissues and collagen fibers in mice were observed under an inverted microscope. The percentage of fibrosis was determined as the blue area divided by the area of the entire field.

Immunohistochemical staining. The kidney tissue sections were deparaffinized and antigen retrieval was performed using a microwave oven at high temperature $(300 \mathrm{~W})$ for $30 \mathrm{~min}$. Following the exhaustion of endogenous peroxidase with methanol and hydrogen peroxide for $30 \mathrm{~min}$ at room temperature, the slides were blocked with $0.5 \%$ bovine serum albumin (Sigma-Aldrich; Merck KGaA) for $60 \mathrm{~min}$ at $37^{\circ} \mathrm{C}$ and incubated with TAK1 antibody (1:200 dilution; cat. no. ab109526; Abcam) overnight at $4^{\circ} \mathrm{C}$. The SABC staining kit (cat. no. SA1026; Boster Biological Technology) was used to perform the chromogenic reaction. Following 
Table I. Exercise training protocol.

\begin{tabular}{lccc}
\hline Week & Session time (min) & Rotations/min & Length (m) \\
\hline 1 & 15 & 16 & 48 \\
2 & 30 & 16 & 96 \\
3 & 30 & 20 & 120 \\
4 & 45 & 20 & 180 \\
5 & 60 & 20 & 240 \\
6 & 60 & 24 & 288 \\
\hline
\end{tabular}

rinsing in PBS, the samples were incubated with the mouse anti-rabbit antibody (cat. no. SA1026; Boster Biological Technology) at $37^{\circ} \mathrm{C}$ for $30 \mathrm{~min}$, followed by rinsing in PBS three times for $5 \mathrm{~min}$. The samples were covered in a drop of DAB developing solution, cell nuclei were stained with hematoxylin at room temperature for $5 \mathrm{~min}$, following dehydration with an alcohol gradient, the samples were cleared with dimethylbenzene xylene and mounted with neutral gum sealant. Photomicrographs of different fields of view were captured using an Olympus DSX100 optical microscope (Olympus Corporation; magnification, $\mathrm{x} 400$ ). and the average optical density (OD) was calculated using Image-pro plus 6.0 analysis software (Media Cybernetics, Inc.).

Immunofluorescence staining. The immunohistochemical staining steps were followed until incubation with the primary antibodies. The samples were incubated with Beclin 1 (1:100 dilution; cat. no. 3738; Cell Signaling Technology, Inc.) or LC3 (1:100 dilution; cat. no. 3868; Cell Signaling Technology, Inc.) overnight at $4^{\circ} \mathrm{C}$. Following thorough washing with PBS, the samples were incubated with fluorescently labeled mouse anti-rabbit (1:2,000 dilution; cat. no. sc-516248; Santa Cruz Biotechnology, Inc.) at $37^{\circ} \mathrm{C}$ for $3 \mathrm{~h}$ (protected from light) and then rinsed in PBS three times for $5 \mathrm{~min}$. The cell nuclei were stained with Hoechst (cat. no. sc-200908; Santa Cruz Biotechnology, Inc.) at room temperature for $5 \mathrm{~min}$, and following mounting with a drop of antifade solution, images were captured with a laser scanning confocal microscope. Image-pro plus 6.0 image analysis software (Media Cybernetics, Inc.) was used to automatically analyze the images and calculate the average optical density.

Western blot analysis. The kidney tissues were obtained by homogenization in a tissue protein extraction reagent supplemented with complete protease and phosphatase inhibitors. The lysate was centrifuged $(12,000 \mathrm{xg})$ at $4^{\circ} \mathrm{C}$ for $5 \mathrm{~min}$ and the supernatant was used. The total protein concentrations were determined using a bicinchoninic acid protein assay kit (Thermo Fisher Scientific, Inc). Protein buffer and $8 \%$ glycerin were added to degenerate the protein for $10 \mathrm{~min}$, and equal quantities of protein ( $40 \mu \mathrm{g} /$ lane) were resolved by $10 \%$ SDS-PAGE, followed by transferred onto polyvinylidene difluoride (PVDF) membranes with a transfer apparatus (Bio-Rad Laboratories, Inc.). The PVDF membranes were incubated in 5\% nonfat milk containing Tris-buffered saline containing Tween-20 (TBST) for $3 \mathrm{~h}$ at room temperature. The PVDF membranes were sequentially incubated with primary antibodies against MKK3 (cat. no. 8535; Cell Signaling Technology, Inc.), phosphorylated (p)-MKK3 (cat. no. 12280; Cell Signaling Technology, Inc.), p38 MAPK (cat. no. ab31828; Abcam), p-p38MAPK (cat. no. ab4822; Abcam) and GAPDH (cat. no. AF0006; Shanghai Boyun Biotech Co., Ltd.) at a dilution of 1:1,000 overnight at $4^{\circ} \mathrm{C}$. Following incubation with primary antibody, the membranes were washed in a TBST four times for $10 \mathrm{~min}$ and incubated in mouse anti-rabbit IgG (H\&L)-horseradish peroxidase conjugate (1:1,500 dilution; cat. no. sc2357; Santa Cruz Biotechnology, Inc.) for $3 \mathrm{~h}$ at room temperature. The blots were visualized using Enhanced Chemiluminescence Western Blotting Luminol Reagent (GE Healthcare) and were semi-quantified using Image-Pro Plus 6.0 image analysis software (Media Cybernetics, Inc.).

Transmission electron microscopy. The kidney tissue was evaluated by autophagosome screening under a JEM-1010 transmission electron microscope (Matsunaga Manufacturing, Co., Ltd., Gifu, Japan). The tissue samples were fixed with $1 \%$ $\mathrm{OsO}_{4}$ in PBS ( $\mathrm{pH} 7.0$ ) for $2 \mathrm{~h}$ and washed three times in PBS, and were then dehydrated with a series of ethanol concentrations for 15-min intervals. The kidney tissues were infiltrated with propylene oxide, and embedded in a mixture of pure Araldite 502 resin and acetone at room temperature for $24 \mathrm{~h}$, $48^{\circ} \mathrm{C}$ for $48 \mathrm{~h}$ and $60^{\circ} \mathrm{C}$ for $48 \mathrm{~h}$, and then cut into $50-60 \mathrm{~nm}$ sections. Finally, the sections were cut into ultrathin sections, and observed with a transmission electron microscope.

Statistical analysis. Statistical analysis of experimental results was performed using GraphPad Prism 5 (GraphPad Software, Inc.), ImageJ 1.8.0 (National Institutes of Health), Image-Pro Plus 6, and SPSS 22.0 (IBM Corp.) statistical software. All data are expressed as the mean \pm SD. Differences among groups were analyzed by one-way analysis of variance followed by Tukey's post hoc test using SPSS 22.0. $\mathrm{P}<0.05$ was considered to indicate a statistically significant difference.

\section{Results}

Incremental load training ameliorates pathological changes of kidney tissue and reduces collagen deposition in aged mice. Masson's staining is the most classical method of collagen fiber staining, with regions of blue staining representing collagen fiber. Microscopic observation revealed normal glomerular morphology and tubular structure in the YC group; furthermore, almost no collagen fibers were produced. In the OC group, glomerular atrophy, an increased level of cytolysis, decreased gaps between the junctions of renal tubular epithelial cells, thickening of the renal tubular wall and deposition of collagen fibers were observed. In the OY group, glomerular atrophy was observed, although the renal tubule walls were not thickened and there was no obvious tubulointerstitial fibrosis (Fig. 1A). Collagen deposition was significantly increased in the OC group compared with that in the $\mathrm{YC}$ group $(\mathrm{P}<0.01)$ and, compared with that in the OC group collagen deposition was significantly decreased following incremental load training $(\mathrm{P}<0.01)$ (Fig. 1B). The above results demonstrated that natural aging may significantly increase the deposition of collagen fibers in 

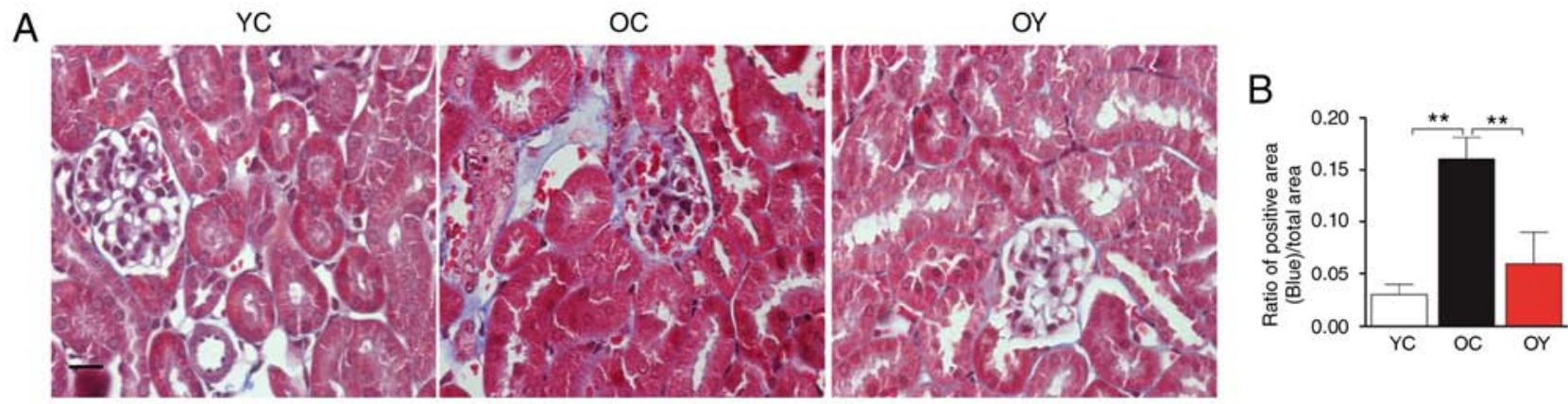

Figure 1. Morphological changes of kidney tissues observed using Masson's staining. (A) Masson staining was used to determine the deposition of collagen in the three groups (magnification, $\mathrm{x} 200$; scale bar=20 $\mu \mathrm{m}$ ). The blue staining represents collagen fibers. (B) Quantitative results showing the degree of fibrosis between the groups. The values represent the mean \pm standard deviation $(\mathrm{n}=5)$. ${ }^{* *} \mathrm{P}<0.01$. YC, young control group; OC, elderly control group; OY, elderly exercise group.

kidney tissues and that incremental load training may reduce the deposition of collagen fibers.

Incremental load training postpones EMT changes in aged mice. E-cadherin and $\alpha$-SMA are markers of mesenchymal changes in renal tubular epithelial cells, therefore, their expression in renal tissue reflects the degree of fibrosis. Western blot analysis was used to assess the protein levels of E-cadherin and $\alpha$-SMA (Fig. 2A). The bar graph suggests that the expression levels of E-cadherin in the OC group were lower than those in the YC group $(\mathrm{P}<0.01)$, whereas the expression levels of $\alpha$-SMA in the OC group were significantly higher than those in the $\mathrm{YC}$ group $(\mathrm{P}<0.01)$. Following incremental load training, the expression levels of E-cadherin in the OY group were significantly higher $(\mathrm{P}<0.05$; Fig. $2 \mathrm{~B})$ and the expression levels of $\alpha$-SMA were significantly lower than those in the OC group $(\mathrm{P}<0.01$; Fig. $2 \mathrm{C})$.

The analysis of immunofluorescence under confocal laser scanning microscopy indicated that, in the YC group, E-cadherin-positive signals were present in the renal tubular epithelium and vascular wall, and a lower level of expression was observed in the renal tubular junction; in the OC group, only a slight E-cadherin-positive signal was present in the renal tubular epithelium. In the OY group, the E-cadherin-positive signals were weaker than those in the $\mathrm{YC}$ group but higher than those in the OC group (Fig. 3A). The quantitative analysis suggested that the OD value of the E-cadherin-positive signal in the OC group was significantly lower than that in the YC group $(\mathrm{P}<0.01)$, and incremental load training significantly increased the OD value of the E-cadherin-positive signal, which differed significantly compared with that in the OC group $(\mathrm{P}<0.01$; Fig. 3B), indicating that incremental load training increased epithelial cell surface markers. However, $\alpha$-SMA exhibited an opposite trend, in the YC group, $\alpha$-SMA was only expressed at low levels in the renal tubular epithelium and vascular wall. In the OC group, the $\alpha$-SMA-positive signal was high; there were uniform, strong positive signals in the renal tubular epithelium and vascular wall, and $\alpha$-SMA was also expressed at low levels in the renal tubular junctions and the wall of the renal capsule. In the OY group, the $\alpha$-SMA-positive signals in the above-mentioned regions were weaker than those in the OC group, but stronger than those in the YC group (Fig. 3A). The quantitative analysis suggested that the OD value of the
$\alpha$-SMA-positive signal was significantly higher than that in the YC group $(\mathrm{P}<0.01)$, and incremental load training significantly reduced the OD value of the $\alpha$-SMA-positive signal, which differed significantly from that in the OC group $(\mathrm{P}<0.01)$, indicating that incremental load training reduced fibroblast surface markers (Fig. 3C).

According to the above experimental results, it can be concluded that incremental load training inhibits the EMT of renal tubular epithelial cells by increasing the expression of epithelial surface marker E-cadherin and decreasing the expression of fibroblast surface marker $\alpha$-SMA. Subsequently, the specific molecular mechanisms were investigated. Previous studies have suggested that the TGF- $\beta 1 /$ TAK1/MKK3/p38MAPK pathway is closely linked to the occurrence and development of EMT. Changes in the expression of these signaling molecules reflect the development of renal fibrosis.

Incremental load training decreases the activity of the TGF- $\beta 1 / T A K 1 / M K K 3 / p 38$ MAPK signaling pathway. Western blot analysis was used to assess the protein levels of TGF- $\beta 1$ and TAK1 (Fig. 4A). The quantitative analysis suggested that the expression levels of TGF- $\beta 1$ and TAK 1 in the OC group were higher than those in the $\mathrm{YC}$ group $(\mathrm{P}<0.01)$. Following incremental load training, the expression levels of TGF- $\beta 1$ and TAK1 in the OY group were significantly lower than those in the OC group $(\mathrm{P}<0.05$; Fig. $4 \mathrm{~B}$ and $\mathrm{C})$. Immunohistochemical staining indicated that the TAK1-positive signal was weak in the YC group. In the OC group, the TAK1-positive signal was strong and was located in the mesangial cells and renal tubular epithelial cells. In the OY group, the TAK1-positive signals in the above-mentioned regions were weaker than those in the OC group, but were stronger than those in the YC group (Fig. 4D). The OD value of the TAK1-positive signal was significantly higher than that in the YC group $(\mathrm{P}<0.01)$. Incremental load training significantly reduced the OD value of the TAK1-positive signal, which differed significantly compared with that in the OC group $(\mathrm{P}<0.01$; Fig. 4E).

Western blot analysis of MKK3, p-MKK3, p38MAPK and p-p38MAPK was then performed (Fig. 5A). The quantitative analysis indicated that the levels of p-MKK3/MKK3 $(\mathrm{P}<0.01)$ and p-p38 MAPK/p38 MAPK $(\mathrm{P}<0.05)$ in the OC group were significantly higher than those in the $\mathrm{YC}$ group, whereas 
A

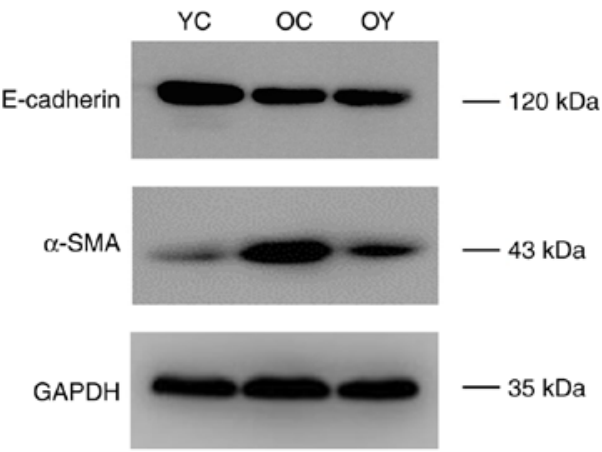

B

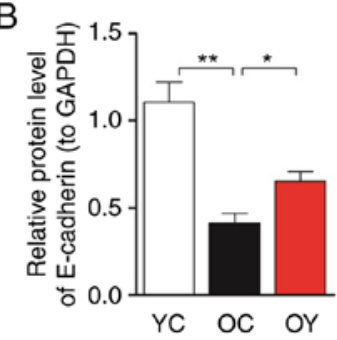

$\mathrm{C}$

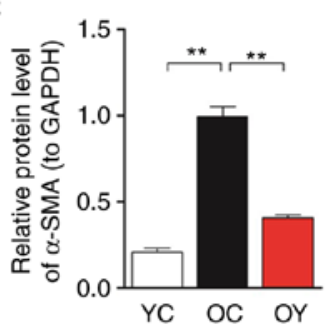

Figure 2. Effects of incremental load training on the expression of E-cadherin and $\alpha$-SMA in kidney tissues of aged mice. (A) Western blot analysis was used to determine the protein expression of E-cadherin and $\alpha$-SMA in the three groups. Bar graphs show the relative quantification of (B) E-cadherin and (C) $\alpha$-SMA. Values are expressed as the mean \pm standard deviation $(n=3) . ~ P<0.05,{ }^{* *} \mathrm{P}<0.01$. YC, young control group; OC, elderly control group; OY, elderly exercise group; $\alpha$-SMA, $\alpha$-smooth muscle actin.

A

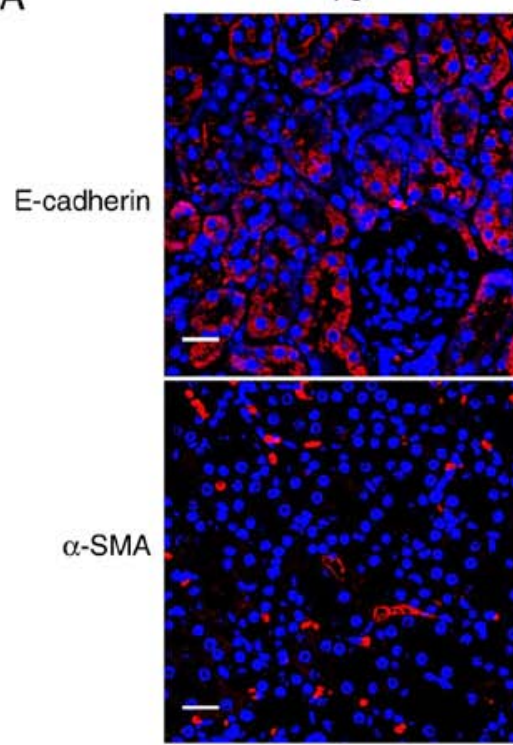

OY

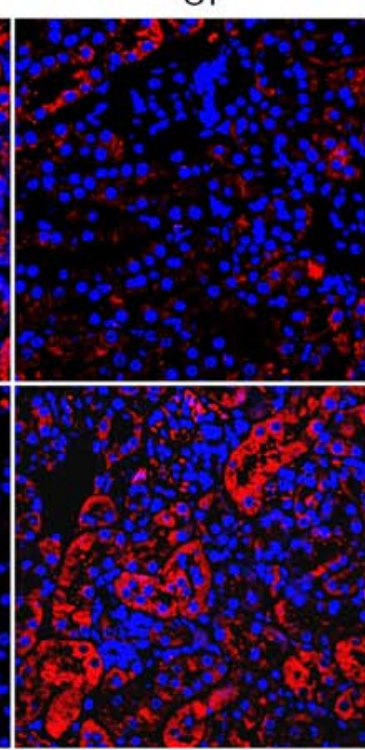

$\mathrm{OC}$

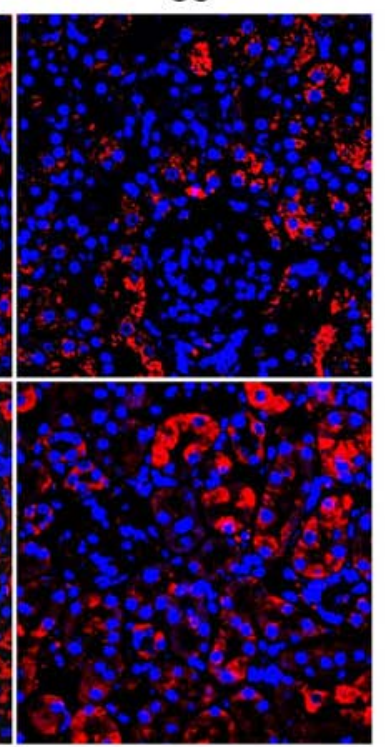

B

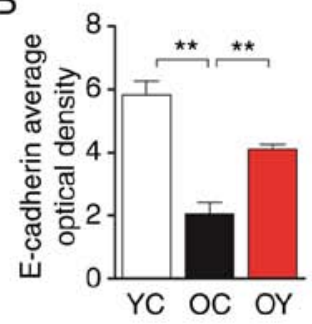

C

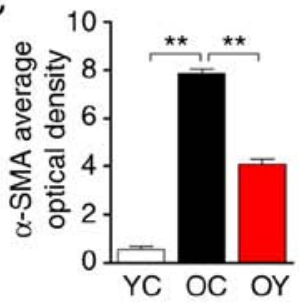

Figure 3. Effects of incremental load training on the expression of the E-cadherin and $\alpha$-SMA in the kidney tissues of aged mice. (A) Expression of E-cadherin and $\alpha$-SMA were identified by immunofluorescence staining (magnification, $\mathrm{x} 400$; scale bar $=20 \mu \mathrm{m}$ ). The red color represents a positive signal; cell nuclei were counterstained with Hoechst (blue). Relative OD values of (B) E-cadherin and (C) $\alpha$-SMA staining are shown in bar graphs. Values are expressed as the mean \pm standard deviation $(n=3) .{ }^{* *} \mathrm{P}<0.01$. YC, young control group; OC, elderly control group; OY, elderly exercise group; OD, optical density; $\alpha$-SMA, $\alpha$-smooth muscle actin.

incremental load training significantly reduced the levels of p-MKK3/MKK3 and p-p38MAPK/p38MAPK compared with those in the $\mathrm{OC}$ group $(\mathrm{P}<0.05$; Fig. 5B and $\mathrm{C})$.

Incremental load training enhances autophagy markers Beclin 1 and LC3. The results of the RT-sqPCR analysis suggested that the mRNA expression levels of Beclin 1 $(\mathrm{P}<0.01)$ and LC3 $(\mathrm{P}<0.05)$ in the OC group were lower than those in the YC group, whereas incremental load training significantly increased the mRNA levels of Beclin $1(\mathrm{P}<0.01)$ and LC3 $(\mathrm{P}<0.05)$ compared with those in the OC group (Fig. 6A-C). The immunofluorescence staining indicated that, in the YC group, the signal of Beclin 1 was high and uniform, and positive signals were observed in the basal region of the renal tubular epithelium in addition to the renal capsule wall and vascular wall. In the OC group, the positive signal of Beclin 1 was weak, and Beclin 1 was uniformly expressed at low levels in the renal tubular epithelium. In the OY group, the overall expression in the above-mentioned regions was higher than that in the OC group (Fig. 6D). The quantitative analysis indicated that the OD value of the Beclin 1-positive signal in the OC group was significantly lower than that in the YC group $(\mathrm{P}<0.01$; Fig. $6 \mathrm{E})$, whereas incremental load training significantly increased the OD value of the Beclin 1-positive signal compared with that in the $\mathrm{OC}$ group $(\mathrm{P}<0.01$; Fig. 6E). In the YC group, the LC3-positive signals were observed in the renal tubular epithelium and vascular wall, and a low level of positive expression was present in the renal tubular junction; in the OC group, LC3 was only expressed at low levels in the renal tubular epithelium. In the OY group, the LC3-positive signals were weaker than those in the YC group, but stronger than those in the OC group (Fig. 6D). The quantitative analysis suggested that the OD value of the LC3-positive signal in the OC group was significantly lower 
A

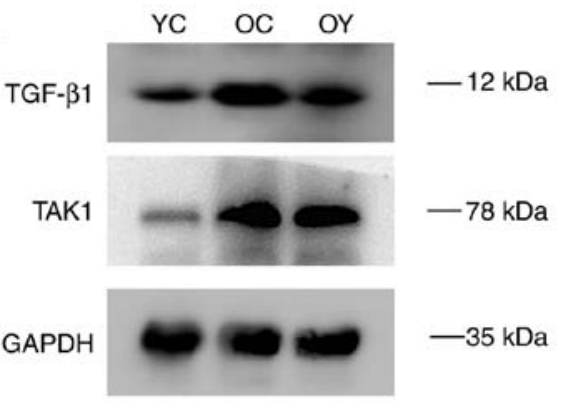

B

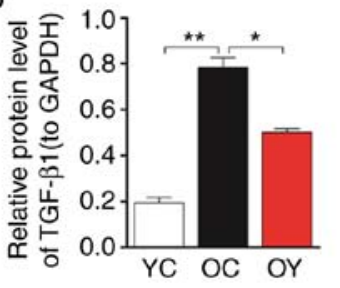

C

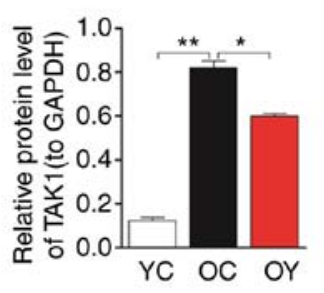

$\mathrm{D}$

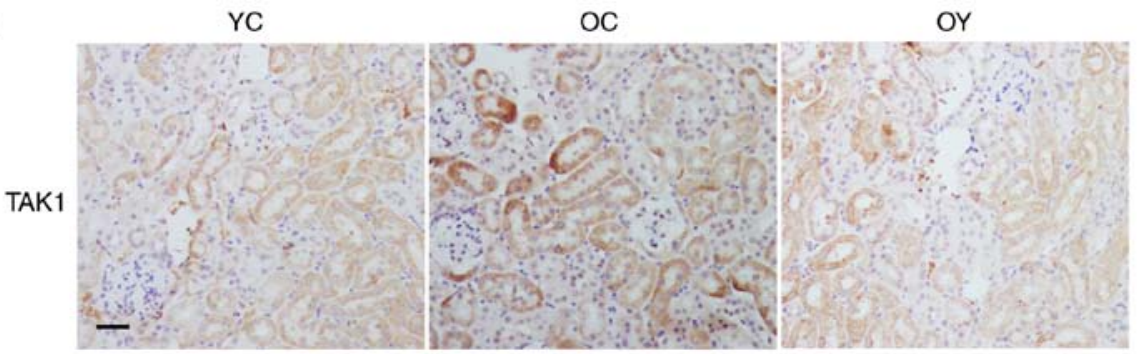

$E$

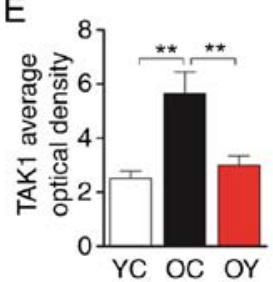

Figure 4. Effects of incremental load training on the expression of TGF- $\beta 1$ and TAK1 in the kidney tissues of aged mice. (A) Western blot analysis was used to determine the protein expression of TGF- $\beta 1$ and TAK1 in the three groups. Bar graphs show the relative quantification of (B) TGF- $\beta 1$ and (C) TAK1. (D) Expression of TAK1 was assessed by immunohistochemical staining (magnification, $\mathrm{x} 200$; scale bar=20 $\mu \mathrm{m}$ ). The brown color indicates a positive signal. (E) Relative OD values of TAK1 staining are shown in a bar graph. Values are expressed as the mean \pm standard deviation ( $\mathrm{n}=3$ ). ${ }^{*} \mathrm{P}<0.05$, ${ }^{* *} \mathrm{P}<0.01$. $\mathrm{YC}, \mathrm{young}$ control group; OC, elderly control group; OY, elderly exercise group; TGF, transforming growth factor; OD, optical density; TAK1, TGF- $\beta$-activated kinase 1 .

A

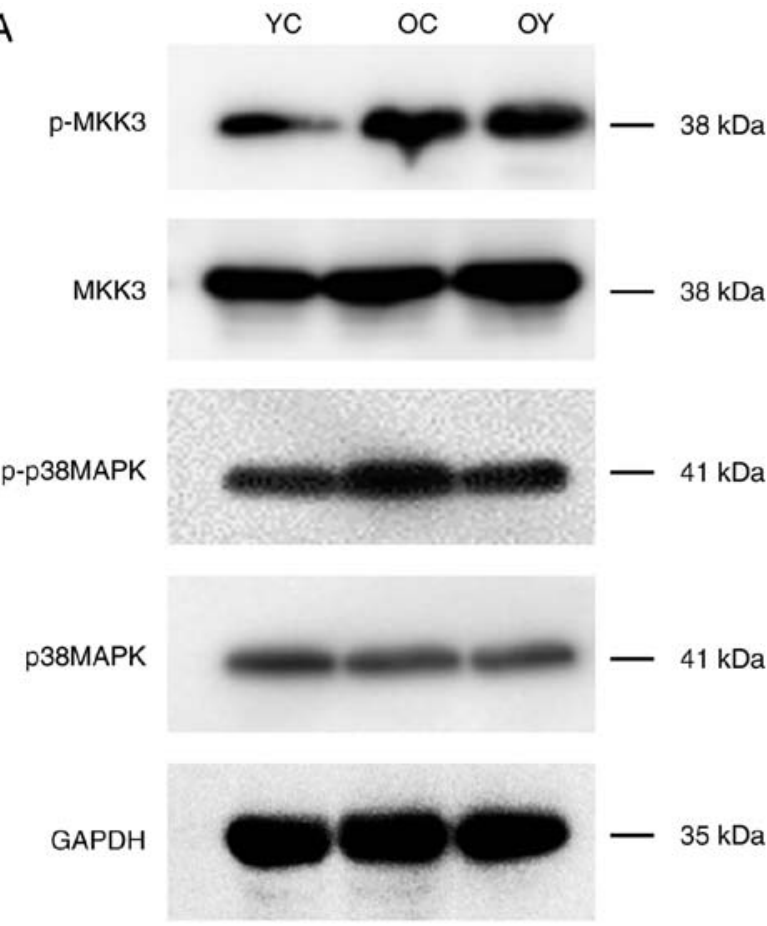

B
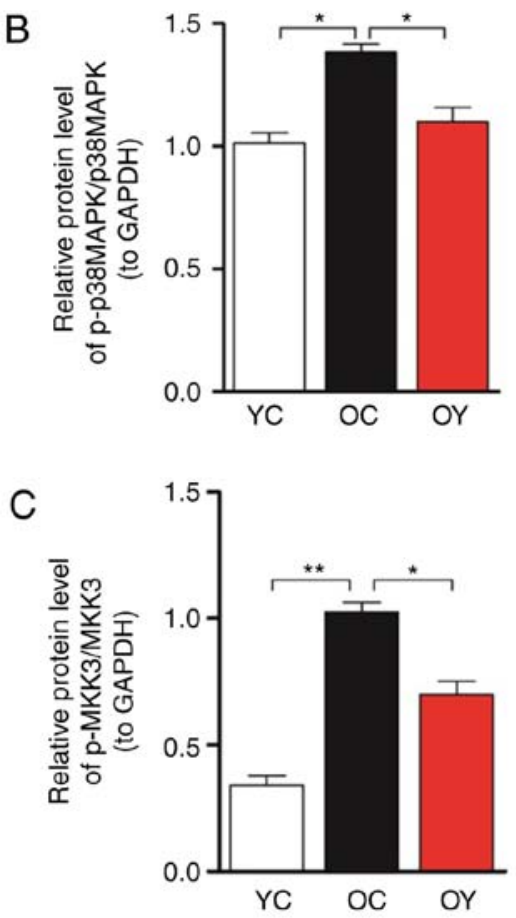

Figure 5. Effects of incremental load training on the expression of MKK3, p-MKK3 p38MAPK and p-p38MAPK in the kidney tissues of aged mice. (A) Western blot analysis was used to determine changes in the protein expression of p-MKK3/MKK3 and p-p38MAPK/p38MAPK in the three groups. Bar graphs show the relative quantification of (B) p-MKK3/MKK3 and (C) p-p38MAPK/p38MAPK. Values are expressed as the mean \pm standard deviation (n=3). ${ }^{*} \mathrm{P}<0.05,{ }^{* *} \mathrm{P}<0.01$. YC, young control group; OC, elderly control group; OY, elderly exercise group; MAPK, mitogen-activated protein kinase; MKK, MAPK kinase; p-, phosphorylated.

than that in the $\mathrm{YC}$ group $(\mathrm{P}<0.01)$, whereas incremental load training significantly increased the OD value of the LC3-positive signal compared with that in the OC group $(\mathrm{P}<0.01$; Fig. 6F). In the YC group, an increased number of autophagosomes with typical bilayer membrane morphology was observed by transmission electron microscopy. The numbers of intracellular autophagosomes in the OY group was increased compared with that in the OC group, indicating that incremental load training increased autophagic activity (Fig. 6G). 
A

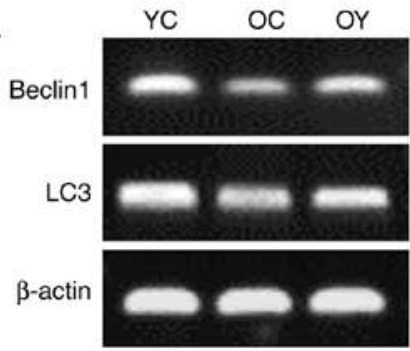

B

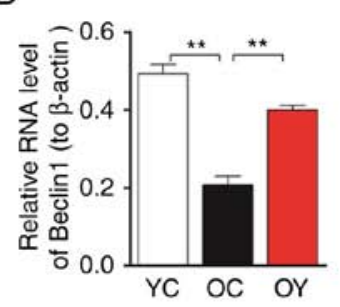

C

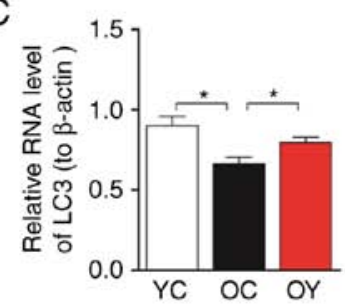

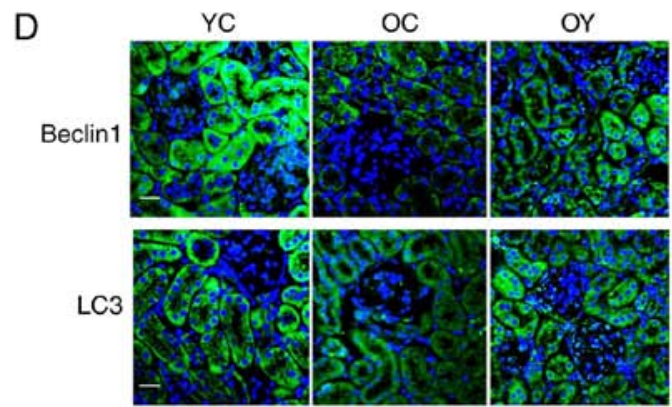
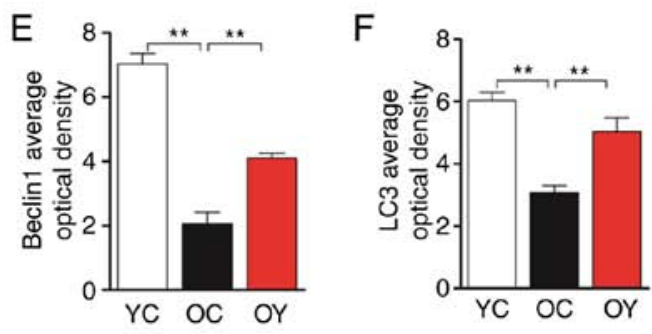

G

YC

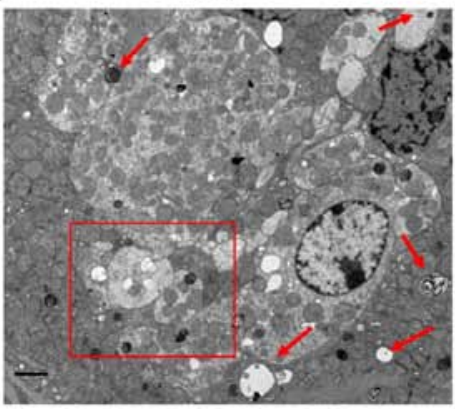

OC

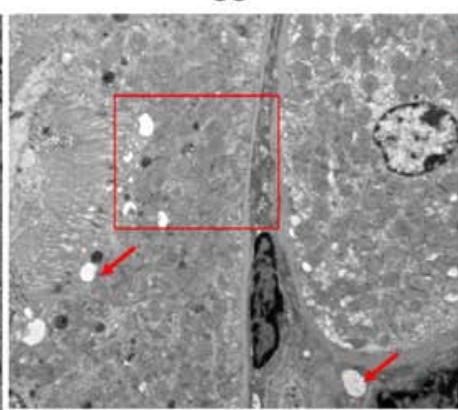

OY
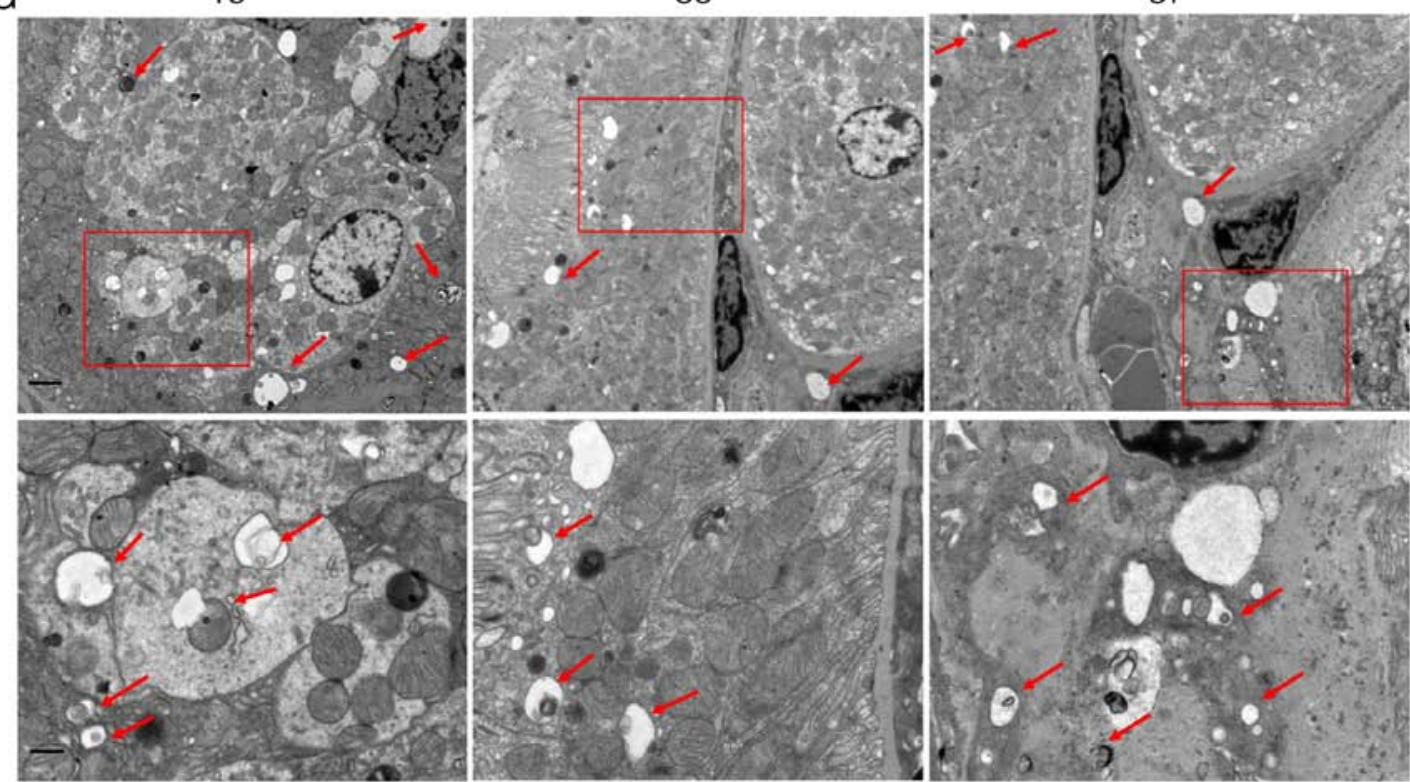

Figure 6. Effects of incremental load training on autophagic markers Beclin 1 and LC3 in the kidney tissues of aged mice. (A) Comparison of the effect of incremental load training on the relative mRNA expression of Beclin 1 and LC3 in kidney tissues. Bar graphs show the relative quantification of (B) Beclin 1 and (C) LC3 mRNA. (D) Expression of Beclin 1 and LC3 was assessed by immunofluorescence staining (magnification, $\mathrm{x} 200$; scale bar=20 $\mu \mathrm{m}$ ). The green color indicates the positive signal; cell nuclei were counterstained with DAPI (blue). Bar graphs show the relative OD values of (E) Beclin1 and (F) LC3 staining. Values are expressed as the mean \pm standard deviation $(n=3) .{ }^{*} \mathrm{P}<0.05,{ }^{* *} \mathrm{P}<0.01$. (G) Assessment of autophagic activity by transmission electron microscopy (magnification, x6,000 and x15,000; scale bars, 2 and $1 \mu \mathrm{m}$ ). The arrows indicate autophagic vacuoles. YC, young control group; OC, elderly control group; OY, elderly exercise group; OD, optical density; LC3, light chain 3.

\section{Discussion}

The present study indicated the presence of slight pathological structural changes and collagen deposition in naturally aged kidney tissues. Decreases in the epithelial surface marker E-cadherin and the autophagic markers Beclin 1 and LC3, and an increase of the fibroblast surface marker $\alpha$-SMA were also observed. Incremental load training was shown to inhibit the TGF- 1 1/TAK1/MKK3/P38MAPK signaling pathway and enhance autophagic activity, to further reduce collagen deposition, and to indirectly upregulate E-cadherin and downregulate
$\alpha$-SMA, thereby improving renal fibrosis induced by collagen deposition in aged mice.

With the advent of an aging population, health problems caused by aging and age-related diseases have become increasingly prominent. The results of an epidemiological survey indicated that diseases linked to renal aging have become a threat to the health and life of middle-aged and elderly individuals (23). A large number of studies have shown that aged kidney tissues exhibit notable fibrosis $(24,25)$. Sangaralingham et al (26) performed Sirius red staining on the renal tissues of rats of different ages, and the results indicated 
that, as age increased, the deposition of collagen fibers in the cortex and medulla increased significantly. Ning et al (27) performed Masson's staining and PAS staining to reveal that, compared with a young age group, the glomerular basement membrane and renal tubular wall in an older age group were thickened and fibrosis was present. In the present study, Masson's staining was used to observe 2-month-old and 19-month-old mice, and the results suggested the presence of glomerular atrophy, increased cytolysis and marked deposition of collagen fibers in the aged mice, indicating fibrosis in the kidney tissues of the aged mice, which is consistent with the results of previous studies.

Exercise is one of the effective rehabilitation methods to delay aging, which may delay the aging of skeletal muscle and brain tissue (28-30). Previous studies have indicated that exercise may also improve glomerular filtration function and glomerulosclerosis in obese individuals (31). Furthermore, the effect of exercise on improving renal fibrosis caused by disease in models has been demonstrated. Peng et al (32) subjected rats with chronic kidney disease to swimming exercise and revealed that this exercise reduced the expression of $\alpha$-SMA, matrix metalloproteinase-2 and CD34, inhibited the process of epithelial cell transdifferentiation and reduced the deposition of ECM, thereby improving renal fibrosis. However, whether exercise is able to improve the renal fibrosis caused by natural aging has not been reported previously. In the present study, 12 aged mice were trained using rotarod treadmill incremental load training for 6 weeks, and the results demonstrated that the level of cytolysis in the kidney tissues of the exercise group was lower than that in the non-training group and the level of collagen fiber deposition was significantly lower than that in the control group, suggesting that incremental load training is able to delay renal fibrosis in aged mice.

Renal fibrosis is mainly characterized by the excessive deposition of ECM components, and EMT is an important factor leading to renal fibrosis. In this process, epithelial cells initiate the reprogramming of gene expression, which is manifested by a decrease in epithelial surface marker E-cadherin and increase in fibroblast surface marker $\alpha$-SMA. In a rat model of renal fibrosis, El-Wakeel et al (33) demonstrated that the expression of E-cadherin decreased and the expression of $\alpha$-SMA increased significantly. Similar results were also obtained in rat models of natural aging-associated renal fibrosis and of ureteral occlusion renal fibrosis $(34,35)$. The results of the immunofluorescence analysis in the present study also indicated that the positive signals of $\alpha$-SMA in the YC group were weak, and that $\alpha$-SMA was uniformly expressed at low levels in the renal tubular epithelium; furthermore, the positive signal of $\alpha$-SMA in the OC group was strong, and numerous positive signals were observed in the renal tubular epithelium, also being expressed in the wall of the renal capsule. However, the positive signal of E-cadherin exhibited an opposite trend, suggesting that incremental load training may delay EMT, reduce the deposition of collagen fibers and improve renal fibrosis in aged mice.

Autophagy is a conserved process of cell component degradation and cycling depending on lysosomes that eliminates damaged organelles and abnormal proteins to maintain a stable intracellular environmental and stress response (36). To date, numerous basic and clinical studies have reported on the critical function of autophagy in the occurrence and development of fibrosis $(37,38)$. Ceylan-Isik et al $(37)$ indicated that aging-induced cardiac interstitial fibrosis is closely linked to the decrease of autophagic activity. Histological examination revealed that interstitial fibrosis in aged mice was significantly aggravated, with a reduction of autophagic markers Beclin-1, autophagy related (Atg)7, Atg 5 and LC3. TGF- $\beta 1$ is a multipotent cytokine that has been established as a central mediator of kidney fibrosis (39). TGF- $\beta 1$ is closely linked to the occurrence and development of autophagy, which results in a decrease of autophagic flux (40). TAK1 is one of the MKK kinases that is involved in TGF- $\beta$ signal transduction in TGF- $\beta 1$-induced type I collagen and fibronectin expression through the activation of MKK3/p38 (41). Kim et al (42) demonstrated that the TAK1/MKK3/p38 signaling pathway mediated the induction of autophagy by TGF- $\beta 1$, controlling the level of type I collagen. In the present study, the immunohistochemical analysis of TAK1 indicated that numerous positive signals were present in the renal tubular epithelium in the OC group, and TAK1 was also expressed in the wall of the renal capsule; however, in the YC group, limited positive signals were observed in the renal tubular epithelium. The results of the western blot analysis indicated that the levels of TGF- $\beta 1$, TAK1, p-MKK3 and p-p38MAPK in the OC group were significantly higher than those in the YC group. Furthermore, the levels were decreased following incremental load training. The above results suggest that incremental load training is able to improve renal fibrosis in aged mice by regulating the TGF- $\beta 1 / \mathrm{TAK} 1 / \mathrm{MKK} 3 / \mathrm{p} 38$ MAPK signaling pathway, enhancing autophagic activity and the expression of Beclin 1 and LC3, increasing the expression of E-cadherin, downregulating the expression of $\alpha$-SMA and reducing collagen deposition, thus improving aging-associated renal fibrosis.

In conclusion, the present study first indicated that incremental load training, as an important rehabilitation intervention, was able to delay renal fibrosis in aged mice, and its mechanism is may linked to inhibition of the TGF- $\beta 1 / \mathrm{TAK} 1 / \mathrm{MKK} 3 / \mathrm{p} 38 \mathrm{MAPK}$ signaling pathway, increases in the expression of Beclin 1 and LC3, the downregulation of $\alpha$-SMA and the upregulation of E-cadherin. However, the direct effects of incremental load training on the TGF- $\beta 1 / \mathrm{TAK} 1 / \mathrm{MKK} 3 / \mathrm{p} 38 \mathrm{MAPK}$ signaling pathway in delaying renal fibrosis in aged mice remains uncertain. In particular, the further use of TGF- $\beta 1$ inhibitors is required to determine the direct effect of the TGF- $\beta 1 / \mathrm{TAK} 1 / \mathrm{MKK} 3 / \mathrm{p} 38 \mathrm{MAPK}$ signaling pathway on renal fibrosis in aged mice.

\section{Acknowledgements}

Not applicable.

\section{Funding}

This study was financially supported by the National Natural Science Foundation of China (grant nos. 31500969 to HL and 31471148 to ZY). 


\section{Availability of data and materials}

The datasets used and/or analyzed during the current study are available from the corresponding author on reasonable request.

\section{Authors' contributions}

BS and HLL contributed equally to this study; HLL conceived and designed the experiments; CCB performed the experiments; QL analyzed the data; QYC prepared figures; ZY collected the data, and edited and revised the manuscript; all authors read and approved the final version of the manuscript.

\section{Ethics approval and consent to participate}

All surgical and care procedures were approved by the Laboratory Animal Welfare and Ethics Committee of the Third Military Medical University (Chongqing, China).

\section{Patient consent for publication}

Not applicable.

\section{Competing interests}

The authors declare that they have no competing interests.

\section{References}

1. Liu Y: Renal fibrosis: New insights into the pathogenesis and therapeutics. Kidney Int 69: 213-217, 2006.

2. Yang $\mathrm{HC}$ and Fogo AB: Fibrosis and renal aging. Kidney Int Suppl 4: 75-78, 2014.

3. Wang B, Xu M, Li W, Li X, Zheng Q and Niu X: Aerobic exercise protects against pressure overload-induced cardiac dysfunction and hypertrophy via $\beta 3-A R-n N O S-N O$ activation. PLoS One 12 e0179648, 2017.

4. Zeina $\mathrm{K}$ and Jennifer T: Anatomic and physiologic changes of the aging kidney. Clin Geriatr Med 29: 555-564, 2013.

5. Stone RC, Pastar I, Ojeh N, Chen V, Liu S, Garzon KI and Tomic-Canic M: Epithelial-mesenchymal transition in tissue repair and fibrosis. Cell Tissue Res 365: 495-506, 2016.

6. Hinz B, Phan SH, Thannickal VJ, Galli A, Bochaton-Piallat ML and Gabbiani G: The myofibroblast: One function, multiple origins. Am J Pathol 170: 1807-1816, 2007.

7. Harikrishna T, Xu XC, Polosukhin VV, Degryse AL, Li B, Han W, Sherrill TP, Plieth D, Neilson EG, Blackwell TS and Lawson WE: Contribution of epithelial-derived fibroblasts to bleomycin-induced lung fibrosis. Am J Respir Crit Care Med 180 657-665, 2009

8. Fîlfan M, Sandu RE, Zăvăleanu AD, GreșiŢă A, Glăvan DG, Olaru DG and Popa-Wagner A: Autophagy in aging and disease. Rom J Morphol Embryol 58: 27-31, 2017.

9. Samy L, Jian X and Rik D: Molecular mechanisms of epithelialmesenchymal transition. Nat Rev Mol Cell Biol 15: 178-196, 2014.

10. Biernacka A, Dobaczewski $M$ and Frangogiannis NG: TGF- $\beta$ signaling in fibrosis. Growth Factors 29: 196-202, 2011.

11. Ding Y, Kim SL, Lee SY, Koo JK, Wang Z and Choi ME: Autophagy regulates TGF- $\beta$ expression and suppresses kidney fibrosis induced by unilateral ureteral obstruction. J Am Soc Nephrol 25: 2835-2846, 2014.

12. Islam SS, Mokhtari RB, El Hout Y, Azadi MA, Alauddin M, Yeger $\mathrm{H}$ and Farhat WA: TGF- $\beta 1$ induces EMT reprogramming of porcine bladder urothelial cells into collagen producing fibroblasts-like cells in a Smad2/Smad3-dependent manner. J Cell Commun Signal 8: 39-58, 2014.

13. Kaur A, Riaz M, Singh SK and Kishore U: Human surfactant protein D suppresses epithelial-to-mesenchymal transition in pancreatic cancer cells by downregulating TGF- $\beta$. Front Immunol 15: 1844, 2018.
14. Kajino T, Omori E, Ishii S, Matsumoto $K$ and Ninomiya-Tsuji J: TAK1 MAPK kinase kinase mediates transforming growth factor-beta signaling by targeting SnoN oncoprotein for degradation. J Biol Chem 282: 9475-9481, 2007.

15. Choi ME, Ding Y and Kim SI: TGF- $\beta$ signaling via TAK1 pathway: Role in kidney fibrosis. Semin Nephrol 32: 244-252, 2012.

16. Li J, Chen K, Li S, Feng J, Liu T, Wang F, Zhang R, Xu S, Zhou Y, Zhou S, et al: Protective effect of fucoidan fromFucus vesiculosuson liver fibrosis via the TGF- $\beta 1 /$ Smad pathway-mediated inhibition of extracellular matrix and autophagy. Drug Des Devel Ther 10: 619-630, 2016.

17. Kim SI, Na HJ, Ding Y, Wang Z, Lee SJ and Choi ME: Autophagy promotes intracellular degradation of type I collagen induced by transforming growth factor (TGF)- $\beta 1$. J Biol Chem 287: 11677-11688, 2012.

18. Banks L, Buchan TA and Dizonno V: Aerobic exercise attenuates ageing of the athletic heart. J Physiol 594: 3183-3184, 2016.

19. Inoue A, Cheng XW, Huang Z, Hu L, Kikuchi R, Jiang H, Piao L, Sasaki T, Itakura $\mathrm{K}, \mathrm{Wu} \mathrm{H}$, et al: Exercise restores muscle stem cell mobilization, regenerative capacity and muscle metabolic alterations via adiponectin/AdipoR1 activation in SAMP10 mice. J Cachexia Sarcopenia Muscle 8: 370-385, 2017.

20. Baker EJ and Gleeson TT: The effects of intensity on the energetics of brief locomotor activity. J Exp Biol 202: 3081-3087, 1999.

21. Bedford TG, Tipton CM, Wilson NC, Oppliger RA and Gisolfi CV: Maximum oxygen consumption of rats and its changes with various experimental procedures. J Appl Physiol Respir Environ Exerc Physiol 47: 1278-1283, 1979.

22. Di Felice V, Macaluso F, Montalbano A, Gammazza AM, Palumbo D, Angelone T, Bellafiore M and Farina F: Effects of conjugated linoleic acid and endurance training on peripheral blood and bone marrow of trained mice. J Strength Cond Res 21: 193-198, 2007.

23. O'Sullivan ED, Hughes J and Ferenbach DA: Renal aging: Causes and consequences. J Am Soc Nephrol 28: 407-420, 2017.

24. Hou CL, Wang MJ, Sun C, Huang Y, Jin S, Mu XP, Chen Y and Zhu YC: Protective effects of hydrogen sulfide in the ageing kidney. Oxid Med Cell Longev 2016: 7570489, 2016.

25. Lin CH, Chen J, Ziman B, Marshall S, Maizel J and Goligorsky MS: Endostatin and kidney fibrosis in aging: A case for antagonistic pleiotropy? Am J Physiol Heart Circ Physiol 306: H1692-H1699, 2014.

26. Sangaralingham SJ, Heublein DM, Grande JP, Cataliotti A, Rule AD, McKie PM, Martin FL and Burnett JC Jr: Urinary C-type natriuretic peptide excretion: A potential novel biomarker for renal fibrosis during aging. Am J Physiol Renal Physiol 301: 943-952, 2011.

27. Ning YC, Cai GY, Zhuo L, Gao JJ, Dong D, Cui S, Feng Z, Shi SZ, Bai XY, Sun XF and Chen XM: Short-term calorie restriction protects against renal senescence of aged rats by increasing autophagic activity and reducing oxidative damage. Mech Ageing Dev 134: 570-579, 2013.

28. Reyes DRA, Gomes MJ, Rosa CM, Pagan LU, Zanati SG, Damatto RL, Rodrigues EA, Carvalho RF, Fernandes AAH, Martinez PF, et al: Exercise during transition from compensated left ventricular hypertrophy to heart failure in aortic stenosis rats. J Cell Mol Med 23: 1235-1245, 2019.

29. Zhou CN, Chao FL, Zhang Y, Jiang L, Zhang L, Luo YM, Xiao Q, Chen LM and Tang Y: Sex differences in the white matter and myelinated fibers of APP/PS1 mice and the effects of running exercise on the sex differences of AD mice. Front Aging Neurosci 10: 243, 2018.

30. Kou X, Li J, Liu X, Chang J, Zhao Q, Jia S, Fan J and Chen N: Swimming attenuates D-galactose-induced brain aging via suppressing miR-34a-mediated autophagy impairment and abnormal mitochondrial dynamics. J Appl Physiol (1985) 122: 1462-1469, 2017.

31. Martínez R, Kapravelou G, López-Chaves C, Cáceres E, Coll-Risco I, Sánchez-González C, Llopis J, Arrebola F, Galisteo M, Aranda P, et al: Aerobic interval exercise improves renal functionality and affects mineral metabolism in obese Zucker rats. Am J Physiol Renal Physiol 316: F90-F100, 2019.

32. Peng CC, Chen KC, Hsieh CL and Peng RY: Swimming exercise prevents fibrogenesis in chronic kidney disease by inhibiting the myofibroblast transdifferentiation. PLoS One 7: e37388, 2012.

33. El-Wakeel SA, Rahmao RM and EI-Abhar HS: Anti-fibrotic impact of Carvedilol in a CCl-4 model of liver fibrosis via serum microRNA-200a/SMAD7 enhancement to bridle TGF- $\beta 1 /$ EMT track. Sci Rep 8: 14327, 2018. 
34. Dong D, Cai GY, Ning YC, Wang JC, Lv Y, Hong Q, Cui SY, Fu B, Guo YN and Chen XM: Alleviation of senescence and epithelial-mesenchymal transition in aging kidney by short-term caloric restriction and caloric restriction mimetics via modulation of AMPK/mTOR signaling. Oncotarget 8: 16109-16121, 2017.

35. Qi FH, Cai PP, Liu X and Si GM: Adenovirus-mediated P311 ameliorates renal fibrosis through inhibition of epithelial-mesenchymal transition via TGF- 31 -Smad-ILK pathway in unilateral ureteral obstruction rats. Int J Mol Med 41: 3015-3023, 2018.

36. De Rechter S, Decuypere JP, Ivanova E, van den Heuvel LP, De Smedt H, Levtchenko E and Mekahli D: Autophagy in renal diseases. Pediatr Nephrol 31: 737-752, 2016.

37. Ceylan-Isik AF, Dong M, Zhang Y, Dong F, Turdi S, Nair S, Yanagisawa $M$ and Ren J: Cardiomyocyte-specific deletion of endothelin receptor A rescues aging-associated cardiac hypertrophy and contractile dysfunction: Role of autophagy. Basic Res Cardiol 108: 335, 2013.

38. Yang S, Abdulla R,Lu C and Zhang L: Inhibition of microRNA-376b protects against renal interstitial fibrosis via inducing macrophage autophagy by upregulating Atg 5 in mice with chronic kidney disease. Kidney Blood Press Res 43: 1749-1764, 2018.
39. Ding $Y$ and Choi ME: Regulation of autophagy by TGF- $\beta$ : Emerging role in kidney fibrosis. Semin Nephrol 34: 62-71, 2014.

40. Sosulski ML, Gongora R, Danchuk S, Dong C, Luo F and Sanchez CG: Deregulation of selective autophagy during aging and pulmonary fibrosis: The role of TGF 31 . Aging Cell 14: 774-783, 2015.

41. Ono K, Ohtomo T, Ninomiya-Tsuji J and Tsuchiya M: A dominant negative TAK1 inhibits cellular fibrotic responses induced by TGF- $\beta$. Biochem Biophys Res Commun 307: 332-337, 2003.

42. Kim SI, Kwak JH, Zachariah M, He Y, Wang L and Choi ME TGF-beta-activated kinase 1 and TAK1-binding protein 1 cooperate to mediate TGF-beta1-induced MKK3-p38 MAPK activation and stimulation of type I collagen. Am J Physiol Renal Physiol 292: F1471-F1478, 2007.

This work is licensed under a Creative Commons Attribution-NonCommercial-NoDerivatives 4.0 International (CC BY-NC-ND 4.0) License. 